Meta

Journal des traducteurs

Translators' Journal

\title{
Le texte publicitaire : traduction ou adaptation?
}

\section{Claude Tatilon}

Volume 35, numéro 1, mars 1990

Actes du colloque international " La traduction proligère "

URI : https://id.erudit.org/iderudit/004326ar

DOI : https://doi.org/10.7202/004326ar

Aller au sommaire du numéro

Éditeur(s)

Les Presses de l'Université de Montréal

ISSN

0026-0452 (imprimé)

1492-1421 (numérique)

Découvrir la revue

Citer cet article

Tatilon, C. (1990). Le texte publicitaire : traduction ou adaptation? Meta, 35(1),

243-246. https://doi.org/10.7202/004326ar d'utilisation que vous pouvez consulter en ligne.

https://apropos.erudit.org/fr/usagers/politique-dutilisation/ 


\section{LE TEXTE PUBLICITAIRE : TRADUCTION OU ADAPTATION?}

Claude TATILON

Université York, Toronto, Canada

Nourrissant des intentions franchement mercantiles, le discours publicitaire est un discours de la persuasion. Sa finalité, tout axée sur le déclenchement de gestes de consommation, n'est obscure pour personne. Non moins claire la stratégie que ce discours adopte: elle consiste à traquer le quidam, médiatiquement, dans les recoins de sa vie quotidienne (son écran de télé, les pages de son journal, les murs de sa ville...) pour lui asséner des textes drastiques, dont l'efficacité est, croit-on, proportionnelle à l'attrait de leur expression (qui les dote d'un pouvoir de relaxation) et, surtout, à la séduction de leur contenu (qui les dote de vertus dormitives).

Telle annonce nous promet monts et merveilles :

Alors qu'elle était endettée de 56000 \$, UNE JEUNE MÈRE DIVORCÉE RACONTE COMMENT ELLE EST DEVENUE MILLIONNAIRE EN 34 MOIS... et comment il vous est aujourd'hui possible de gagner assez d'argent chez vous pour prendre votre retraite millionnaire (sic) d'ici 5 à 10 ans - sans effort ${ }^{1}$.

Telle autre nous assure qu'en huit jours, la lotion HYPERTRIX aura stoppé net notre chute de cheveux; qu'un mois plus tard, de jeunes pousses déjà couvriront notre chef; qu'avant l'août, foi d'animal, les fruits passeront la promesse des fleurs et qu'à la saison nouvelle - garanti - le reboisement sera complet ${ }^{2}$ !

Dans le texte publicitaire, les débordements du contenu et de l'expression sont destinés à faciliter l'action persuasive en conduisant à un engourdissement de la vigilance. Et sans doute n'est-il pas rare (si l'on en croit l'énormité des budgets publicitaires) que cette sorte d'agression douce soit agissante, c'est-à-dire que les textes infligés parviennent à investir du statut d'acheteurs bon nombre de leurs destinataires.

Quant à l'écriture caractéristique par laquelle se matérialise un bon texte publicitaire, elle a pour principaux attributs la clarté et l'inattendu. Clarté : pour que rien ne se perde du message ; inattendu : pour secouer la lassitude et le désintérêt engendrés par le harcèlement d'une multitude de messages concurrents. Mais remarquons ici que ces deux propriétés ne sont pas du tout antinomiques. Dans un texte publicitaire, les pirouettes du signifiant n'aboutissent jamais à l'hermétisme tel qu'on peut le rencontrer en poésie, car l'acuité du message en serait alors émoussée. C'est pourquoi les audaces verbales de la publicité restent toujours «cousues de fil blanc».

Ces généralités étant rappelées, imaginons maintenant un modèle descriptif qui nous permette de distinguer clairement les caractéristiques discursives du texte publicitaire et, par là, d'évaluer sa portée commerciale (ou incitative). Ce modèle, je crois pouvoir le ramener à quatre fonctions essentielles : d'abord, deux fonctions manifestées par le contenu - une fonction identificatrice émanant de la désignation de l'objet exhibé et une fonction laudative émanant de l'indication d'une qualité propre à cet objet ; ensuite, deux autres fonctions manifestées par l'expression - une fonction ludique produite par un jeu de langage (souvent venu de l'oral) et une fonction mnémotechnique assumée par la lisibilité et la prégnance de l'annonce ${ }^{3}$. 
Essayons de retrouver ces fonctions dans deux énoncés types dont le texte publicitaire ne saurait se passer: le nom de marque et le slogan. Portons d'abord notre attention sur le nom de marque, aussi indispensable à un produit commercial que l'adresse et le timbre à un envoi postal. Trait essentiel de l'identification, le nom de marque est avant tout un mot-phare, mais il est, de surcroît, la première des armes persuasives de la panoplie publicitaire. En effet, plus que tout autre vocable de la langue, il a vocation de se substituer à la réalité qu'il désigne et d'en incarner les séductions : Coca-Cola fait saliver, Air Canada fait voyager, Cartier rêver, Chanel phantasmer... Tous mots-talismans, dont il est important d'apprécier la force appellative et le rayonnement intratextuel pour mieux comprendre le fonctionnement particulier de la communication publicitaire. Le traducteur, il est vrai, a rarement l'occasion de traduire les noms de marque; mais cela lui est parfois nécessaire. Le shampooing nord-américain Pert, après quelques années de désignation unilingue, est brutalement devenu Prêt dans la version canadienne-française. On comprendra mieux le bien-fondé de l'opération si l'on sait qu'à l'origine le liquide était blanc: Pert (White)! Il est vert aujourd'hui, avec une bonne odeur d'herbe coupée...

Les noms de marque sont dangereux; ils n'en sont que plus précieux. Aussi sont-ils adroitement ciselés, présentés dans de somptueux écrins typographiques. Signés, encartouchés, damasquinés, enrubannés, ensoleillés même, ils connaissent les hauts et les bas de casse, les pleins et les déliés, les maigres et les gras, et nous en font voir de toutes les couleurs. Quelquefois, ils donnent lieu à des larcins. Le très utilitaire Scotch Tape, revêtu d'un tartan, vole un peu de son prestige à la boisson nationale écossaise. Le fromage Kiri, après l'échec commercial d'une vache trop sérieuse, joue le jeu de celle dont le rire persiste. Pepsi-Cola vole à droite et à gauche - à Coca-Cola, d'un côté; de l'autre, à $D^{r}$ Pepper. La guerre des noms de marque fait rage. Dans la mêlée, parfois, d'extraordinaires éclats: le fulgurant Kodak; la mise à feu syllabique d'un Dubonnet, déclenchée par un ingénieux slogan à trois étages, Dubo-Dubon-Dubonnet; la géniale concision de L'eggs. (Il s'agit de bas de nylon vendus dans une boîte en forme d'œuf.) Plein à craquer de force suggestive, ce dernier nom, motivé par la forme de l'emballage ${ }^{4}$, mérite qu'on s'y attarde un instant: beauté naturelle de l'œuf à l'ovale parfait (fonction laudative), qui fait jeu de mots, en anglais, avec les jambes (fonction ludique, voire érotique), tout en évoquant, par son article inattendu, la francité et, par association, la haute couture parisienne (de nouveau, fonction laudative). Nos quatre fonctions en une seule syllabe: qui dit mieux?

Donnons-nous maintenant un peu plus de tissu textuel et examinons un second type d'énoncé - le slogan ${ }^{5}$ - omniprésent lui aussi dans le texte publicitaire. Comme le prouve ce «rédactionnel», dont deux phrases sur cinq ${ }^{6}$ sont des slogans.

\section{LE BORDEAUX ME VA BIEN}

Parce qu'il me ressemble, le Bordeaux... Rouge ou blanc, sec ou liquoreux, il est de toutes mes humeurs, et toujours élégant. Le savoir boire, c'est aussi du savoir vivre. Alors c'est sûr, le Bordeaux me va bien.

\section{BORDEAUX, LA COULEUR DU BON GOÛT}

Nos quatre fonctions y sont bien présentes. Comme elles le sont, à l'évidence, dans cet autre slogan: On a toujours besoin de petits pois chez soi! Très réussi quant à l'écriture, jouant sur un intertexte reçu cinq sur cinq par le grand public, cet énoncé a pourtant mal tourné en France où, après quelques mois de matraquage, on constata une baisse sensible dans les ventes des légumes en conserve. Explication avancée par les experts: les petits pois avaient malencontreusement éveillé dans l'esprit des gens le souvenir des temps difficiles de l'Occupation allemande, du marché noir, du stockage du sucre... Les voies de la Publicité sont souvent impénétrables! 
Mais terminons sur une note moins négative avec un autre slogan français, qui n'a pas encore été retiré de la circulation (malgré une faute d'orthographe). C'est celui qui agrémente un paquet de tampons à récurer vendus par deux, sous le label Scotch-Brite. (Encore le scotch! Et tant mieux si le nom est étranger: le produit n'en paraîtra que plus nouveau.) Son slogan, très concis, est irrésistible: 2 gratton (sic)-laveurs. Rien que pour cette astucieuse trouvaille, les sympathiques créatures verbales mériteraient de faire beaucoup de mousse.

Après cette analyse fonctionnelle, qui a permis de décrire ce que je crois être la dynamique de la communication publicitaire en mettant au jour (sauf erreur) les quatre ressorts principaux de sa force de persuasion, la consigne traductologique qui me semble s'imposer est de traduire non la lettre mais l'esprit, non les mots mais les fonctions. Traduction relâchée donc, réduite à l'essentiel - l'équivalence fonctionnelle - et qu'on a sans doute intérêt à désigner d'un autre nom, adaptation me paraissant convenir tout à fait. Le modèle fonctionnel proposé devrait alors rendre service, à deux moments décisifs de l'opération traduisante: au tout début, lorsqu'il s'agit d'apprécier le texte de départ à sa juste valeur, d'en sélectionner l'information pertinente; et à la toute fin, lorsqu'il s'agit de vérifier l'exactitude, la «fidélité» du texte d'arrivée ${ }^{7}$.

Nous savons tous que l'information d'un texte n'est pas toujours transférable dans sa totalité d'une langue-culture à une autre langue-culture. Juste avant moi, Françoise Wuilmart a montré, avec beaucoup de finesse, que certains contenus de nature littéraire voyagent mal. Mais elle l'a fait sans pessimisme, il est important de le faire remarquer. En effet, si l'on théorise dans le sens d'une conservation «pure et dure» des contenus, force est alors de constater que certains ne passent pas. Une telle ligne de raisonnement ne peut que conduire à des repentirs, à des conclusions défaitistes sur la nature de l'opération traduisante. Personnellement, je préfère croire en une traductologie militante qui, cernant bien les problèmes, ne les résout sans doute pas tous mais s'applique, du moins, à déculpabiliser la pratique, à la favoriser. C'est ce que cherche à faire la stratégie de l'équivalence des fonctions, dont j'ai trouvé l'idée chez Eugene A. Nida ${ }^{8}$ et surtout chez Georges Mounin. Cette démarche réaliste permet d'éliminer bien des cas dits d'kintraduisibilité» - et ainsi de «traduire sans trahir», pour reprendre le titre du beau livre de JeanClaude Margot ${ }^{9}$. Ou encore, empruntant le terme à André Martinet, je dirai que ce genre d'équivalence permet, dans certains cas particulièrement difficiles, d'effectuer une «repensée» des messages en les adaptant aux «sucs gastriques» des consommateurs du texte d'arrivée. (Vous reconnaissez, cette fois, une image de Françoise Wuilmart.)

Ainsi, par exemple, un slogan français affirmant l'élégance d'un parapluie, $I l$ vous plaira avant même qu'il ait plu, gardera toute sa vigueur commerciale s'il devient en anglais: When it rains, it reigns (en jouant sur l'homophonie des verbes «pleuvoir» et «régner» dans cette langue). Ou, pour en revenir au domaine alimentaire et faire oublier l'échec cuisant des petits pois hexagonaux, on pourra proposer de traduire (pardon, d'adapter) Tea. Iced'n easy par T'as chaud? Thé glacé! Belle réussite québécoise, qui mériterait bien d'être signée. Ou enfin, ce titre: Poulet au vinaigre (marque de commerce, en quelque sorte, d'un produit qui est ici un film policier français). Fricoté à l'américaine, ce poulet français devient, par un vrai miracle culinaire, un savoureux Coq au vin ${ }^{10}$ !

\section{NOTES}

1. Cette annonce-conte de fées occupe deux pleines pages (pp. 28-29) du supplément Télé + du quotidien montréalais La Presse du samedi 16 septembre 1989

2. Texte inventé - quelque peu caricatural, je l'admets.

3. À ce propos, voir mon article intitulé «Traduire la parole publicitaire» (La Linguistique, 1978, 14-1, Paris, pp. 76-87). 
4. L'aspect iconique des annonces publicitaires, qui par ailleurs mérite toute l'attention du traductologue, sort évidemment des limites du présent exposé. J'ai récemment abordé ce sujet dans une communication intitulée Le Texte imagé, dans laquelle j'essayais de déterminer la part respective du textuel et du visuel dans la production du sens (Congrès des sociétés savantes du Canada, Association canadienne de traductologie, Université Laval, 28-30 mai 1989).

5. À consulter, d'Olivier REBOUL : Le Slogan (Paris, PUF, 1975) et «Le slogan et les fonctions du langage» (Le Fançais dans le monde, 143, février-mars 1979, pp. 21-26).

6. Cinq: syntaxiquement parlant. Ne nous arrêtons pas ici à la ponctuation «morcelante», qui fait usage d'un procédé de mise en relief (imitation écrite de la forme orale du langage) courant mais toujours efficace.

7. Sur l'équivalence fonctionnelle, voir la $4^{\mathfrak{e}}$ partie, «Considérations méthodologiques», de mon Traduire. Pour une pédagogie de la traduction (Toronto, Éditions du GREF, 1987).

8. Nida, après avoir longtemps parlé de dynamic equivalence, terme peu clair, a adopté celui de functional equivalence dans son dernier ouvrage (Jan de WAARD \& Eugene A. NIDA, From One Language to Another, New York, Thomas Nelson Publishers, 1986).

9. Jean-Claude MARGOT, Traduire sans trahir, Lausanne, L'Âge d'Homme, 1979.

10. Exemple cité par Jean-Paul BRUNET dans sa communication présentée, en mai dernier, à l'Université Laval (voir ci-dessus note 4). 